International Journal of Computer Science \& Information Technology (IJCSIT) Vol 4, No 2, April 2012

\title{
Signal Processing for MIMO Interference Networks
}

\author{
Thanat Chiamwichtkun ${ }^{1}$, Stephanie Soon ${ }^{2}$ and Ian $\mathrm{Lim}^{3}$ \\ ${ }^{1}$ Bangkok University, Thailand \\ ${ }^{2,3}$ National University of Singapore, Singapore
}

\begin{abstract}
Multiple Input Multiple Output Interference Channels are investigated in this paper. We focus on how to tackle the interference when different users try to send their codewords to their corresponding receivers. We propose a strategy to remove the interference while allowing different users transmit at the same time. Our strategy is low-complexity while the performance is good. Mathematical analysis is provided and simulations are given based on our system.
\end{abstract}

\section{KEYWORDS}

MIMO, Interference Channel, Alamouti Codes, Diversity, Interference Cancellation, Complexity.

\section{INTRODUCTION}

Multiple antennas, when used at both the transmitter and the receiver, create a multiple-input multiple-output (MIMO) propagation channel. Using sophisticated coding at the transmitter and substantial signal processing at the receiver, the MIMO channel can be provisioned for higher data rates, resistance to multipath fading, lower delays, and support for multiple users. [17].Current research efforts demonstrate that MIMO technology has great potential in third and fourth-generation $(3 \mathrm{G}, 4 \mathrm{G})$ cellular systems, fixed wireless access, wireless local area networks, and ad hoc wireless battlefield networks. Optimizing MIMO networks using channel state information at the transmitter (often called closed-loop MIMO communication) can help customize the transmitted waveforms to provide higher link capacity and throughput, improve system capacity by sharing the spatial channel with multiple users simultaneously, enable channel- aware scheduling for multiple users, simplify multi-user receivers through interference avoid avoidance, and provide a simple and general means to exploit spatial diversity. Essentially, channel state information makes it easier to obtain the benefits of MIMO technology while lessening the complexity impact incurred through MIMO transmission and reception [8-35].

A consequence of using multiple antennas, however, is an increase in the number of channel state parameters. Training can be used to estimate the channel at the receiver. In some cases the transmit channel can be inferred from the receive channel, but more often channel state information needs to be quantized and sent to the transmitter over a limited rate feedback channel. This is not unreasonable; control channels are often available to implement power control, adaptive modulation, and certain closed-loop diversity modes

DOI : $10.5121 /$ ijcsit.2012.4209 


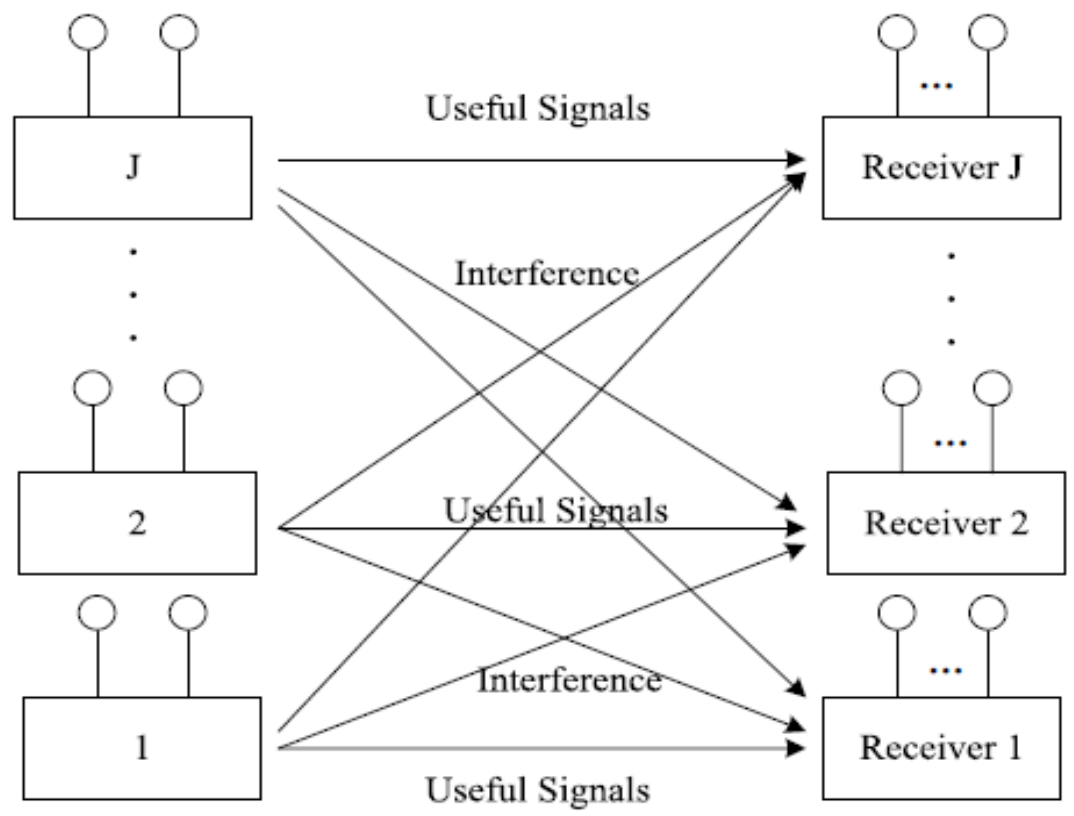

Figure 1: Channel Model

(e.g., in 3G). Unfortunately, the feedback requirements in a MIMO system generally grow with the product of the transmit antennas, receive antennas, delay spread, and number of users, while the capacity only grows linearly.

An interference channel is a network consisting $N$ senders and $N$ receivers. There exists a oneto-one correspondence between senders and receivers. Each sender only wants to communicate with its corresponding receiver, and each receiver only cares about the information form its corresponding sender. However, each channel interferes the others. So an interference channel has $\mathrm{N}$ principal links and $N(N-1)$ interference links. This scenario often occurs, when several sender-receiver pairs share a common media. For example, in satellite communication, two satellites send information to its corresponding ground station simultaneously. Each ground station can receive the signals from both of the two satellites and its communication is interfered by the other pair's communication. The study of this kind of channel was initiated by Shannon in 1961. However, this channel has not been solved in general case even in the general Gaussian case.

In this paper, we focus on MIMO interference channels [8-14]. Since each user transmits at the same time, how to deal with the co-channel interference is an interesting question. Schemes to cancel the co-channel interference when channel knowledge is known at the transmitter are proposed in [15-19]. In this paper, we propose and analyze a scheme when channel knowledge is not known at the transmitter, a scenario which is more practical. The article is organized as follows. In the next section the system model is introduced. Detailed interference cancellation procedures are provided and performance analysis is given. Then simulation results are presented. Concluding remarks are given in the final section. 
International Journal of Computer Science \& Information Technology (IJCSIT) Vol 4, No 2, April 2012

\section{Interference Cancellation and Performance Analysis}

Assume there are $J$ transmitters each with 2 transmit antennas and $J$ receivers each equipped with $J$ receive antennas. Each transmit sends codewords to different receivers. So this is an interference channel. Let $c_{t, n}(j)$ denote the transmitted symbol from the $n$-th antenna of user $j$ at transmission interval $t$ and $r_{t, m}$ be the received word at the receive antenna $m$ at the receiver. Then, for the received symbols we will have

$$
r_{t, m}=\sum_{j=1}^{J} \sum_{n=1}^{N} \alpha_{n, m}(j) c_{t, n}(j)+\eta_{t, m}
$$

It is well-known that one can separate signals sent from $J$ different users each equipped with $N$ transmit antennas, with enough receive antennas. We can simply form a decoding matrix that is orthogonal to the space spanned by channel coefficients of the users to be eliminated. For example, if we let

$$
\begin{gathered}
R_{t}=C_{t} H+N_{t} \\
H(j)=\left(\begin{array}{cccc}
\alpha_{1,1}(j) & \alpha_{1,2}(j) & \cdots & \alpha_{1, M}(j) \\
\alpha_{2,1}(j) & \alpha_{2,2}(j) & \cdots & \alpha_{2, M}(j) \\
\vdots & \vdots & \ddots & \vdots \\
\alpha_{N, 1}(j) & \alpha_{N, 2}(j) & \cdots & \alpha_{N, M}(j)
\end{array}\right)
\end{gathered}
$$

where $j$ denotes the $j$ th user. Therefore, one can rewrite Equation (2) as follows:

$$
R_{t}=\sum_{j=1}^{J} C_{t}(j) H(j)+N_{t}
$$

To decode user 1, one can simply find a zero-forcing(ZF) matrix $Z$ such as

$$
H(1) Z \neq 0
$$

and

$$
H(j) Z=0 \text { for } j \neq 1
$$

In other words, $Z$ should null the space spanned by the row vectors of all $H(j) \mathrm{s}$, for $j=2,3, \ldots$, $J$. Also, it should not null at least one row vector of $H(1)$. Since all the rows of $H(j)$ s might be linearly independent, the dimension of $Z$, i.e. $M$, must be at least equal to the number of these rows, or $(J-1) N+1$. Each antenna group (user) can employ a modulation scheme to benefit transmit diversity; as if it is the only group that is sending data.

In order to reduce the number of required receive antennas, we propose a scheme to cancel the interference with less number of receive antennas. 
International Journal of Computer Science \& Information Technology (IJCSIT) Vol 4, No 2, April 2012

Consider $J$ users each transmitting Alamouti code, i.e. Orthogonal Space-Time Block Code (OSTBC) $\left(\begin{array}{cc}a_{1} & a_{2} \\ -a_{2}^{*} & a_{1}^{*}\end{array}\right)$ to a receiver equipped with at least $J$ receive antennas. The received signal at the $i$ th receive antenna of a given receiver with interference can be written in the following format:

$$
\begin{gathered}
\left(\begin{array}{c}
r_{1, i}(k) \\
r_{2, i}(k)
\end{array}\right)=\sum_{j=1}^{J}\left(\begin{array}{cc}
s_{1}(j) & s_{2}(j) \\
-s_{2}(j)^{*} & s_{1}(j)^{*}
\end{array}\right)\left(\begin{array}{c}
\alpha_{1, i}(j, k) \\
\alpha_{2, i}(j, k)
\end{array}\right) \\
+\left(\begin{array}{c}
\eta_{1, i}(k) \\
\eta_{2, i}(k)
\end{array}\right)
\end{gathered}
$$

Here we assume $J$ is the number of receivers with interference and $k$ denotes the $k$ th receiver. The idea behind interference cancellation arises from separate decodability of each symbol; at each receive antenna we perform the decoding algorithm as if there is only one user. This user will be the one the effect of whom we want to cancel out. Then, we simply subtract the softdecoded value of each symbol in one of the receive antennas from the rest and as a result remove the effect of that user. This procedure is presented in the following. For the receiver with interference, at the $i$ th antenna, we have

$$
\begin{array}{r}
\left(\begin{array}{c}
r_{1, i}(k) \\
r_{2, i}(k)^{*}
\end{array}\right)=\sum_{j=1}^{J}\left(\begin{array}{cc}
\alpha_{1, i}(j, k) & \alpha_{2, i}(j, k) \\
\alpha_{2, i}(j, k)^{*} & -\alpha_{1, i}(j, k)^{*}
\end{array}\right) \\
\times\left(\begin{array}{c}
s_{1}(j) \\
s_{2}(j)
\end{array}\right)+\left(\begin{array}{c}
\eta_{1, i}(k) \\
\eta_{2, i}(k)^{*}
\end{array}\right)
\end{array}
$$

For the receiver without interference, at the $i$ th antenna, we have

$$
\begin{aligned}
\left(\begin{array}{c}
r_{1, i}(k) \\
r_{2, i}(k)^{*}
\end{array}\right)= & \left(\begin{array}{cc}
\alpha_{1, i}(j, k) & \alpha_{2, i}(j k) \\
\alpha_{2, i}(j, k)^{*} & -\alpha_{1, i}(j, k)^{*}
\end{array}\right) \\
& \times\left(\begin{array}{c}
s_{1}(j) \\
s_{2}(j)
\end{array}\right)+\left(\begin{array}{c}
\eta_{1, i}(k) \\
\eta_{2, i}(k)^{*}
\end{array}\right)
\end{aligned}
$$


International Journal of Computer Science \& Information Technology (IJCSIT) Vol 4, No 2, April 2012 where $j \in\{1, \ldots, J\}$. In order to cancel the signals $s_{1}$ and $s_{2}$ from User 1 , we first multiply both sides of Equation (8) with $\left(\begin{array}{cc}\alpha_{1, i}(1, k) & \alpha_{2, i}(1, k) \\ \alpha_{2, i}(1, k)^{*} & -\alpha_{1, i}(1, k)^{*}\end{array}\right)^{\dagger}$. Then we have Equations (10):

$$
\begin{gathered}
\left(\begin{array}{cc}
\alpha_{1, i}^{*}(1, k) & \alpha_{2, i}(1, k) \\
\alpha_{2, i}^{*}(1, k) & -\alpha_{1, i}(1, k)
\end{array}\right)\left(\begin{array}{c}
r_{1, i}(k) \\
r_{2, i}(k)^{*}
\end{array}\right) \\
=\left(\left|\alpha_{1, i}(1, k)\right|^{2}+\left|\alpha_{2, i}(1, k)\right|^{2}\right)\left(\begin{array}{c}
s_{1}^{1} \\
s_{2}^{1}
\end{array}\right)+ \\
\left(\begin{array}{cc}
\alpha_{1, i}^{*}(1, k) & \alpha_{2, i}(1, k) \\
\alpha_{2, i}^{*}(1, k) & -\alpha_{1, i}(1, k)
\end{array}\right) \\
\times \sum_{j=2}^{J}\left(\begin{array}{cc}
\alpha_{1, i}(j, k) & \alpha_{2, i}(j, k) \\
\alpha_{2, i}^{*}(j, k) & -\alpha_{1, i}^{*}(j, k)
\end{array}\right)\left(\begin{array}{l}
s_{1}(j) \\
s_{2}(j)
\end{array}\right) \\
+\left(\begin{array}{l}
\eta_{1, i}(k)^{\prime} \\
\eta_{2 . i}(k)^{\prime}
\end{array}\right)
\end{gathered}
$$

where $\eta_{1, i}(k)^{\prime}, \eta_{2, i}(k)^{\prime}$ are given by

$$
\left(\begin{array}{c}
\eta_{1, i}(k)^{\prime} \\
\eta_{2, i}(k)^{\prime}
\end{array}\right)=\left(\begin{array}{cc}
\alpha_{1, i}^{*}(1, k) & \alpha_{2, i}(1, k) \\
\alpha_{2, i}^{*}(1, k) & -\alpha_{1, i}(1, k)
\end{array}\right)\left(\begin{array}{c}
\eta_{1, i}(k) \\
\eta_{2, i}(k)
\end{array}\right)
$$

In order to eliminate the effect of user 1, we need to divide both sides of Equation (10) by

$$
\frac{1}{\left(\left|\alpha_{1, i}(1, k)\right|^{2}+\left|\alpha_{2, i}(1, k)\right|^{2}\right)}
$$

Equations (10) becomes Equations (13):

$$
\begin{gathered}
\frac{1}{\left(\left|\alpha_{1, i}(1, k)\right|^{2}+\left|\alpha_{2, i}(1, k)\right|^{2}\right)} \\
\times\left(\begin{array}{cc}
\alpha_{1, i}^{*}(1, k) & \alpha_{2, i}(1, k) \\
\alpha_{2, i}^{*}(1, k) & -\alpha_{1, i}(1, k)
\end{array}\right)\left(\begin{array}{c}
r_{1, i}(k) \\
r_{2, i}(k)^{*}
\end{array}\right) \\
=\left(\begin{array}{c}
s_{1}^{1} \\
s_{2}^{1}
\end{array}\right)+\frac{1}{\left(\left|\alpha_{1, i}(1, k)\right|^{2}+\left|\alpha_{2, i}(1, k)\right|^{2}\right)}\left(\begin{array}{c}
\eta_{1, i}(k)^{\prime} \\
\eta_{2, i}(k)^{\prime}
\end{array}\right) \\
+\sum_{j=2}^{J} \frac{1}{\left(\left|\alpha_{1, i}(1, k)\right|^{2}+\left|\alpha_{2, i}(1, k)\right|^{2}\right)} \\
\times\left(\begin{array}{cc}
\alpha_{1, i}^{*}(1, k) & \alpha_{2, i}(1, k) \\
\alpha_{2, i}^{*}(1, k) & -\alpha_{1, i}(1, k)
\end{array}\right) \\
\times\left(\begin{array}{cc}
\alpha_{1, i}(j, k) & \alpha_{2, i}(j, k) \\
\alpha_{2, i}^{*}(j, k) & -\alpha_{1, i}^{*}(j, k)
\end{array}\right)\left(\begin{array}{c}
s_{1}(j) \\
s_{2}(j)
\end{array}\right)
\end{gathered}
$$


International Journal of Computer Science \& Information Technology (IJCSIT) Vol 4, No 2, April 2012

Then we can subtract both sides of Equation (13) from the equation when $i=1$. The resulting terms are shown by

$$
\widehat{y}(i, k)=\sum_{j=2}^{J} \widehat{H}(i, k)\left(\begin{array}{l}
s_{1}(j) \\
s_{2}(j)
\end{array}\right)+\left(\begin{array}{l}
\eta_{1, i}(k)^{\prime \prime} \\
\eta_{2, i}(k)^{\prime \prime}
\end{array}\right)
$$

where $\widehat{y}(i, k)$ and $\widehat{H}(i, k), i=2, \ldots, J$, are given by Equations (15) and (16):

$$
\begin{aligned}
& \widehat{y}(i, k)= \\
& \frac{1}{\left|\alpha_{1, i}(1, k)\right|^{2}+\left|\alpha_{2, i}(1, k)\right|^{2}} \\
& \times\left(\begin{array}{cc}
\alpha_{1, i}^{*}(1, k) & \alpha_{2, i}(1, k) \\
\alpha_{2, i}^{*}(1, k) & -\alpha_{1, i}(1, k)
\end{array}\right)\left(\begin{array}{c}
r_{1, i}(k) \\
r_{2, i}(k)^{*}
\end{array}\right) \\
& -\frac{1}{\left|\alpha_{1,1}(1, k)\right|^{2}+\left|\alpha_{2,1}(1, k)\right|^{2}} \\
& \times\left(\begin{array}{cc}
\alpha_{1,1}^{*}(1, k) & \alpha_{2,1}(1, k) \\
\alpha_{2,1}^{*}(1, k) & -\alpha_{1,1}(1, k)
\end{array}\right)\left(\begin{array}{c}
r_{1,1}(k) \\
r_{2,1}(k)^{*}
\end{array}\right)
\end{aligned}
$$

$$
\begin{aligned}
\frac{\hat{H}(i, k)=}{\left|\alpha_{1, i}(1, k)\right|^{2}+\left|\alpha_{2, i}(1, k)\right|^{2}} \\
\times\left(\begin{array}{cc}
\alpha_{1, i}^{*}(1, k) & \alpha_{2, i}(1, k) \\
\alpha_{2, i}^{*}(1, k) & -\alpha_{1, i}(1, k)
\end{array}\right) \\
\times\left(\begin{array}{cc}
\alpha_{1, i}(j, k) & \alpha_{2, i}(j, k) \\
\alpha_{2, i}^{*}(j, k) & -\alpha_{1, i}^{*}(j, k)
\end{array}\right) \\
\left.-\frac{1}{\mid \alpha_{1,1}(1,}, k\right)\left.\right|^{2}+\left|\alpha_{2,1}(1, k)\right|^{2} \\
\times\left(\begin{array}{cc}
\alpha_{1,1}^{*}(1, k) & \alpha_{2,1}(1, k) \\
\alpha_{2,1}^{*}(1, k) & -\alpha_{1,1}(1, k)
\end{array}\right) \\
\times\left(\begin{array}{cc}
\alpha_{1,1}(j, k) & \alpha_{2,1}(j, k) \\
\alpha_{2,1}^{*}(j, k) & -\alpha_{1,1}^{*}(j, k)
\end{array}\right)
\end{aligned}
$$


International Journal of Computer Science \& Information Technology (IJCSIT) Vol 4, No 2, April 2012 $\eta_{1, i}(k)^{\prime \prime}, \eta_{2, i}(k)^{\prime \prime}$ are given by

$$
\begin{aligned}
& \left(\begin{array}{l}
\eta_{1, i}(k)^{\prime \prime} \\
\eta_{2, i}(k)^{\prime \prime}
\end{array}\right) \\
& =\frac{1}{\left(\left|\alpha_{1, i}(1, k)\right|^{2}+\left|\alpha_{2, i}(1, k)\right|^{2}\right)}\left(\begin{array}{l}
\eta_{1, i}(k)^{\prime} \\
\eta_{2, i}(k)^{\prime}
\end{array}\right) \\
& -\frac{1}{\left(\left|\alpha_{1,1}(1, k)\right|^{2}+\left|\alpha_{2,1}(1, k)\right|^{2}\right)}\left(\begin{array}{l}
\eta_{1,1}(k)^{\prime} \\
\eta_{2,1}(k)^{\prime}
\end{array}\right)
\end{aligned}
$$

The distribution of $\eta_{1, i}(k)^{\prime \prime}, \eta_{2, i}(k)^{\prime \prime}$ are Gaussian white noise. In Equation (16), $\hat{H}(i, k)$ can be written as the following structure:

$$
\widehat{H}(i, k)=\left(\begin{array}{cc}
a(i, k) & b(i, k) \\
b(i, k)^{*} & -a(i, k)^{*}
\end{array}\right)
$$

where $a(i, k)$ and $b(i, k)$ are given by Equations (19) and (20):

$$
\begin{aligned}
& a(i)= \\
& \frac{1}{\left|\alpha_{1, i}(1, k)\right|^{2}+\left|\alpha_{2, i}(1, k)\right|^{2}} \\
& \times\left[\alpha_{1, i}^{*}(1, k) \alpha_{1, i}(j, k)+\alpha_{2, i}(1, k) \alpha_{2, i}(j, k)^{*}\right] \\
& -\frac{1}{\left|\alpha_{1,1}(1, k)\right|^{2}+\left|\alpha_{2,1}(1, k)\right|^{2}} \\
& \times\left[\alpha_{1,1}^{*}(1, k) \alpha_{1,1}(j, k)+\alpha_{2,1}(1, k) \alpha_{2,1}^{*}(j, k)\right] \\
& b(i)= \\
& \frac{1}{\left|\alpha_{1, i}(1, k)\right|^{2}+\left|\alpha_{2, i}(1)\right|^{2}} \\
& \times\left[\alpha_{1, i}^{*}(1, k) \alpha_{2, i}(j, k)-\alpha_{2, i}(1, k) \alpha_{1, i}^{*}(j, k)\right] \\
& -\frac{1}{\left|\alpha_{1,1}(1, k)\right|^{2}+\left|\alpha_{2,1}(1, k)\right|^{2}} \\
& \times\left[\alpha_{1,1}^{*}(1, k) \alpha_{2,1}(j, k)-\alpha_{2,1}(1, k) \alpha_{1,1}^{*}(j, k)\right]
\end{aligned}
$$

Till now, we have already cancelled the signals from User 1 . Follow the same procedure, we can cancel the signals from User 2 to User $J-1$. Finally, we can get the signals from User $J$ only as shown below:

$$
\widehat{y}(J, k)=\widehat{H}(J, k)\left(\begin{array}{l}
s_{1}(J) \\
s_{2}(J)
\end{array}\right)+\left(\begin{array}{l}
\eta_{1, J}(k)^{\prime \prime} \\
\eta_{2, J}(k)^{\prime \prime}
\end{array}\right)
$$

In order to decode the $s_{1}(J)$, we can multiply both sides of the Equation (21) with 
International Journal of Computer Science \& Information Technology (IJCSIT) Vol 4, No 2, April 2012

$$
\begin{aligned}
& \left(\begin{array}{c}
a(J, k) \\
b(J, k)^{*}
\end{array}\right)^{\dagger} \text {, we have } \\
& \left.\qquad \begin{array}{c}
a(J, k) \\
b(J, k)^{*}
\end{array}\right)^{\dagger} \widehat{y}(J, k)= \\
& \left(\begin{array}{ll}
|a(J, k)|^{2}+|b(J, k)|^{2} & 0
\end{array}\right)\left(\begin{array}{c}
s_{1}(J) \\
s_{2}(J)
\end{array}\right) \\
& +\left(\begin{array}{c}
a(J, k) \\
b(J, k)^{*}
\end{array}\right)^{\dagger}\left(\begin{array}{l}
\eta_{1, J}(k)^{\prime \prime} \\
\eta_{2, J}(k)^{\prime \prime}
\end{array}\right) \\
& =\left(|a(J, k)|^{2}+|b(J, k)|^{2}\right) s_{1}(J)+ \\
& \left(\begin{array}{c}
a(J, k) \\
b(J, k)^{*}
\end{array}\right)^{\dagger}\left(\begin{array}{l}
\eta_{1, J}(k)^{\prime \prime} \\
\eta_{2, J}(k)^{\prime \prime}
\end{array}\right)
\end{aligned}
$$

In order to keep the Gaussian white noise, we need

$$
\begin{aligned}
& \frac{1}{\sqrt{|a(J, k)|^{2}+|b(J, k)|^{2}}}\left(\begin{array}{c}
a(J, k) \\
b(J, k)^{*}
\end{array}\right)^{\dagger} \widehat{y}(J, k) \\
& =\sqrt{|a(J, k)|^{2}+|b(J, k)|^{2}} s_{1}(J) \\
& +\frac{1}{\sqrt{|a(J, k)|^{2}+|b(J, k)|^{2}}}\left(\begin{array}{c}
a(J, k) \\
b(J, k)^{*}
\end{array}\right)^{\dagger} \\
& \quad \times\left(\begin{array}{l}
\eta_{1, J}(k)^{\prime \prime} \\
\eta_{2, J}(k)^{\prime \prime}
\end{array}\right)
\end{aligned}
$$

Maximum likelihood decoding can be used to decode $s_{1}(J)$ :

$$
\begin{aligned}
& \widehat{s}_{1}(J)= \\
& \arg \min _{s_{1}(J)} \mid \frac{1}{\sqrt{|a(J, k)|^{2}+|b(J, k)|^{2}}}\left(\begin{array}{c}
a(J, k) \\
b(J, k)^{*}
\end{array}\right)^{\dagger} \\
& \times \widehat{y}(J, k)-\left.\sqrt{|a(J, k)|^{2}+|b(J, k)|^{2}} s_{1}(J)\right|_{F} ^{2}
\end{aligned}
$$

So the decoding is symbol-by-symbol. In order to decode the $s_{2}(J)$, we can multiply both sides of the Equation (14) with $\left(\begin{array}{c}b(J, k) \\ -a(J, k)^{*}\end{array}\right)^{\dagger}$, we have 
International Journal of Computer Science \& Information Technology (IJCSIT) Vol 4, No 2, April 2012

$$
\begin{aligned}
& \left(\begin{array}{c}
b(J, k) \\
-a(J, k)^{*}
\end{array}\right)^{\dagger} \widehat{y}(J, k) \\
& =\left(\begin{array}{cc}
0 & |a(J, k)|^{2}+|b(J, k)|^{2}
\end{array}\right)\left(\begin{array}{c}
s_{1}(J) \\
s_{2}(J)
\end{array}\right) \\
& +\left(\begin{array}{c}
b(J, k) \\
-a(J, k)^{*}
\end{array}\right){ }^{\dagger}\left(\begin{array}{l}
\eta_{1, J}(k)^{\prime \prime} \\
\eta_{2, J}(k)^{\prime \prime}
\end{array}\right) \\
& =\left(\begin{array}{c}
\left.|a(J, k)|^{2}+|b(J, k)|^{2}\right) s_{2}(J)+ \\
\left(\begin{array}{c}
b(J, k) \\
-a(J, k)^{*}
\end{array}\right)^{\dagger}\left(\begin{array}{l}
\eta_{1, J}(k)^{\prime \prime} \\
\eta_{2, J}(k)^{\prime \prime}
\end{array}\right)
\end{array}\right.
\end{aligned}
$$

In order to keep the Gaussian white noise, we need

$$
\begin{aligned}
& \frac{1}{\sqrt{|a(J, k)|^{2}+|b(J, k)|^{2}}}\left(\begin{array}{c}
b(J, k) \\
-a(J, k)^{*}
\end{array}\right)^{\dagger} \widehat{y} \\
& =\sqrt{|a(J, k)|^{2}+|b(J, k)|^{2}} s_{2}(J) \\
& +\frac{1}{\sqrt{|a(J, k)|^{2}+|b(J, k)|^{2}}}\left(\begin{array}{c}
b(J, k) \\
-a(J, k)^{*}
\end{array}\right)^{\dagger} \\
& \times\left(\begin{array}{l}
\eta_{1, J}(k)^{\prime \prime} \\
\eta_{2, J}(k)^{\prime \prime}
\end{array}\right)
\end{aligned}
$$

Maximum likelihood decoding can be used to decode $s_{2}(J)$ :

$$
\begin{aligned}
& \widehat{s}_{2}(J)= \\
& \arg \min _{s_{2}(J)} \mid \frac{1}{\sqrt{|a(J, k)|^{2}+|b(J, k)|^{2}}}\left(\begin{array}{c}
b(J, k) \\
-a(J, k)^{*}
\end{array}\right)^{\dagger} \\
& \times \widehat{y}(J, k)-\left.\sqrt{|a(J, k)|^{2}+|b(J, k)|^{2}} s_{2}(J)\right|_{F} ^{2}
\end{aligned}
$$

The decoding is also symbol-by-symbol. Follow the above procedures, we can detect signals with interference. Now we analyze the diversity. From Equation (22), we know that the diversity is determined by factor $\sqrt{|a(J, k)|^{2}+|b(J, k)|^{2}}$. The diversity is defined as

$$
d=-\lim _{\rho \rightarrow \infty} \frac{\log P_{e}}{\log \rho}
$$

where $\rho$ denotes the SNR and $P_{\mathrm{e}}$ represents the probability of error. It is known that the error probability can be written as 
International Journal of Computer Science \& Information Technology (IJCSIT) Vol 4, No 2, April 2012

$$
\begin{aligned}
& P\left(s_{1}(2) \rightarrow \text { error } \mid a, b\right) \\
& =Q\left(\sqrt{\frac{\rho\left|\sqrt{|a(J, k)|^{2}+|b(J, k)|^{2}} \mathbf{e}\right|_{F}^{2}}{4}}\right) \\
& \leq \exp \left(-\frac{\rho\left(|a(J, k)|^{2}+|b(J, k)|^{2}\right) \mathbf{e}^{\dagger} \mathbf{e}}{4}\right) \\
& =\exp \left(-\frac{\rho\left(|a(J, k)|^{2}+|b(J, k)|^{2}\right) e^{2}}{4}\right)
\end{aligned}
$$

where $e$ is the error. We need to analyze $a(J, k)$ and $b(J, k)$. Conditioned on $\alpha_{1,2}(J, k), \alpha_{2,2}(J, k)$, $\alpha_{1,1}(J, k), \alpha_{2,1}(J, k)$, then $a(J, k)$ and $b(J, k)$ are both Gaussian random variables. It is easy to verify that

$$
\begin{aligned}
& E\left[a(J, k) \cdot b(J, k) \mid \alpha_{1,2}(J, k), \alpha_{2,2}(J, k),\right. \\
& \left.\alpha_{1,1}(J, k), \alpha_{2,1}(J, k)\right]=0
\end{aligned}
$$

So $a(J, k)$ and $b(J, k)$ are independent Gaussian random variables. We have

$$
\begin{aligned}
& P\left(s_{1}(2) \rightarrow \text { error }\right) \\
& =E\left[E\left[P\left(s_{1}(2) \rightarrow \text { error } \mid a(J, k), b(J, k)\right)\right] \mid\right. \\
& \left.\quad \alpha_{1,2}(J, k), \alpha_{2,2}(J, k), \alpha_{1,1}(J, k), \alpha_{2,1}(J, k)\right] \\
& \leq E\left[E \left[\exp \left(-\frac{\rho\left(|a(J, k)|^{2}+|b(J, k)|^{2}\right) e^{2}}{4}\right) \mid\right.\right. \\
& =E\left[\frac{\left.\left.\alpha_{1,2}(J, k), \alpha_{2,2}(J), k, \alpha_{1,1}(J, k), \alpha_{2,1}(J, k)\right]\right]}{\left.\prod_{j=1}^{2}\left[1+\frac{\rho e^{2}}{4}\right)\right]} \mid\right. \\
& =\frac{1}{\left.\prod_{j=1}^{2}(J, k), \alpha_{2,2}(J, k), \alpha_{1,1}(J, k), \alpha_{2,1}(J, k)\right]}
\end{aligned}
$$

When $\rho$ is large, Equation (31) becomes

$$
P\left(s_{1}(2) \rightarrow \text { error }\right) \leq \rho^{-2}\left(\frac{e^{2}}{4}\right)^{-2}
$$

By Equation (28), the diversity is 2.

In summary, the interference cancellation based on Alamouti codes can achieve cancel the interference successfully and the decoding complexity is symbol-by-symbol which is the lowest and the diversity is 2, which is the best as far as we know when no channel information is available at the user side and the lowest decoding complexity is required. 
International Journal of Computer Science \& Information Technology (IJCSIT) Vol 4, No 2, April 2012

\section{Simulations}

In order to evaluate the proposed scheme, we use a system with two users with two antennas and two receivers each with two receive antennas. This is a typical interference channel. The two users are sending signals to the receivers simultaneously. We assume alamouti codes are transmitted. So there will be co-channel interference. If the proposed interference cancellation is used, the performance is provided in Figures 2 and 3 while QPSK is used in Figure 2 and 8-PSK is used in Figure 3. In each figure, we compare the interference cancellation scheme with a TDMA scheme with beamforming scheme. That is, during each time slot, one user transmits while the other keeps silent. In order to make the rate the same for the two schemes, in Figure 2, 16-QAM is used while in Figure 3, 64-QAM is used. It is obvious that the proposed scheme has better performance which confirms the effectiveness of the interference cancellation scheme.

\section{Conclusions}

In this paper, we discuss the interference channel. We first give detailed description on interference channel. Later we show that how to tackle interference in such a system is important. Aiming to remove the interference, a strategy for interference channel is proposed and analyzed. The complexity of the strategy is low while the performance is good. Simulations confirm the theoretical analysis.

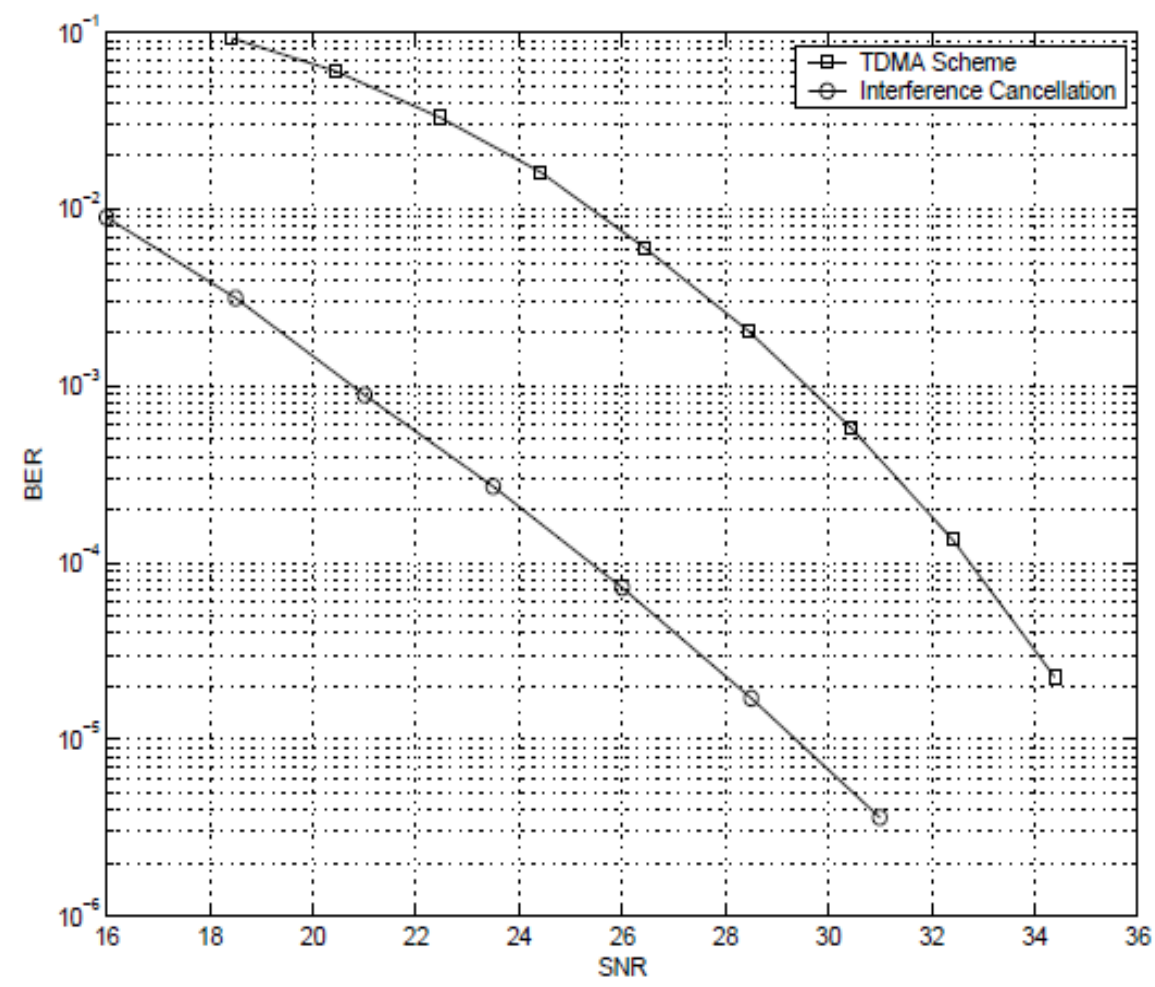

Figure 2: QPSK constellation with interference cancellation 
International Journal of Computer Science \& Information Technology (IJCSIT) Vol 4, No 2, April 2012

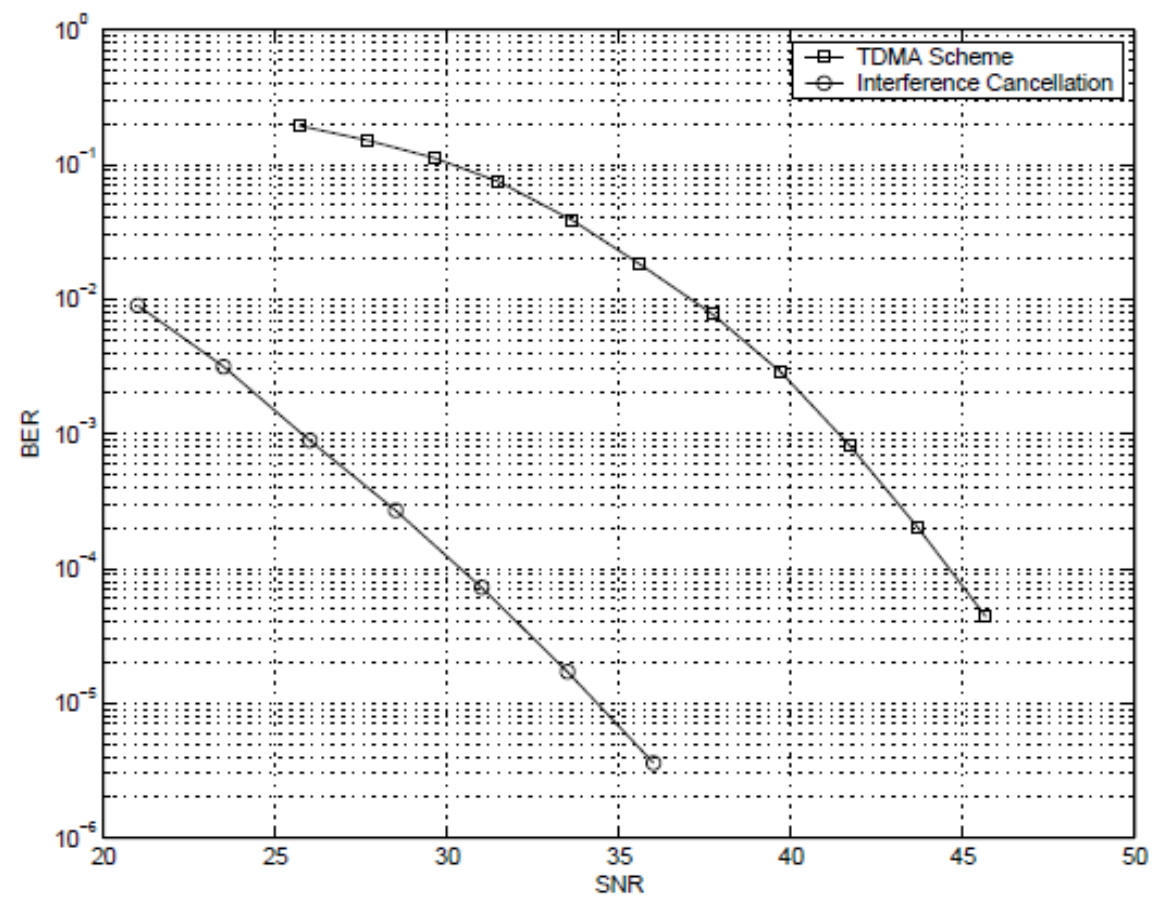

Figure 3: 8-PSK constellation with interference cancellation

\section{REFERENCES}

[1] W. Tarng, H.W. Lin and K. L Ou, "A Cluster Allocation and Routing Algorithm based on Node Density for Extending the Lifetime of Wireless Sensor Networks", International Journal of Computer Science \& Information Technology (IJCSIT), 2012

[2] W. Y. Ge, J. S. Zhang and G. L. Xue, "MIMO-Pipe Modeling and Scheduling for Efficient Interference Management in Multihop MIMO Networks," IEEE Transactions on Vehicular Technology, vol. 59, no. 8, pp. 3966-3978, 2010.

[3] F. Li, "Optimization of input covariance matrix for multi-antenna correlated channels," International Journal of Computer Networks \& Communications (IJCNC), 2011.

[4] F. Li, "Array Processing for Multi-User Multi-Antenna Interference Channels Using Precoders," Wireless Personal Communications, 2012.

[5] C. F. Ball, R. Mullner, J. Lienhart, H. Winkler, "Performance analysis of Closed and Open loop MIMO in LTE," European Wireless Conference, pp. 260-265, 2009.

[6] H. Futaki, T. Ohtsuki, "Low-density parity-check (LDPC) coded MIMO systems with iterative turbo decoding," Vehicular Technology Conference, pp 342-346, 2003.

[7] F. Li and H. Jafarkhani, "Space-Time Processing for X Channels Using Precoders," IEEE Transactions on Signal Processing.

[8] F. Li, Multi-Antenna Multi-User Interference Cancellation and Detection Using Precoders, $\mathrm{PhD}$ thesis, UC Irvine, 2012

[9] A. Goldsmith, S. Jafar, N. Jindal, S. Vishwanath, " Capacity limits of MIMO channels," IEEE Journal on Selected Areas in Communications, vol. 21, no. 5, pp. 684-702, 2003. 
International Journal of Computer Science \& Information Technology (IJCSIT) Vol 4, No 2, April 2012

[10] K. Kusume, G. Dietl, T. Abe, H. Taoka, S. Nagata, "System Level Performance of Downlink MUMIMO Transmission for 3GPP LTE-Advanced," Vehicular Technology Conference, 2010.

[11] F. Li and H. Jafarkhani, "Interference Cancellation and Detection Using Precoders," IEEE International Conference on Communications (ICC 2009), June 2009.

[12] E. M. Mohamed, D. Kinoshita, K. Mitsunaga, Y. Higa, H. Furukawa, "MIMO based wireless backhaul," Ultra Modern Telecommunications and Control Systems and Workshops (ICUMT), 2010.

[13] V. S. Annapureddy, V. V. Veeravalli, "Sum Capacity of MIMO Interference Channels in the Low Interference Regime," IEEE Transactions on Information Theory, vol. 57, no. 5, pp. 2565-2581, 2011.

[14] F. Li and H. Jafarkhani, "Interference Cancellation and Detection for More than Two Users," IEEE Transactions on Communications, March 2011.

[15] S. W. Peters, R. W. Heath, "Cooperative Algorithms for MIMO Interference Channels," IEEE Transactions on Vehicular Technology, vol. 60, no. 1, pp. 206-218, 2011.

[16] A. Chaaban, A. Sezgin, "Interference channel with delay: Noisy interference," International ITG Workshop on Smart Antennas (WSA), 2010.

[17] F. Li and H. Jafarkhani, "Multiple-antenna interference cancellation and detection for two users using quantized feedback," IEEE Transactions on Wireless Communication, vol. 10, no. 1, pp. 154163, Jan 2011.

[18] O. Sahin, O. Simeone, E. Erkip; "Interference Channel With an Out-of-Band Relay," IEEE Transactions on Information Theory, vol. 57, no. 5, pp. 2746-2764, 2011.

[19] F. Li and H. Jafarkhani, "Interference cancellation and detection for multiple access channels with four users," in Proceedings of IEEE International Conference on Communications(ICC 2010), June 2010.

[20] W. Vijacksungsithi, K. A. Winick, "Joint channel-state estimation and decoding of low-density parity-check codes on the two-state noiseless/useless BSC block interference channel," IEEE Transactions on Communications, vol. 53, no. 4, pp. 612-622, 2005.

[21] X. He, A. Yener, "The Gaussian Many-to-One Interference Channel With Confidential Messages," IEEE Transactions on Information Theory, vol. 57, no. 5, pp. 2730-2745, 2011.

[22] F. Li and H. Jafarkhani, "Using quantized feedback to cancel interference in multiple access channels," in Proceedings of IEEE Global Telecommunications Conference(Globecom 2010), December, 2010.

[23] R. Zhang, F. Gao, Y. Liang, “Cognitive beamforming made practical: Effective interference channel and learning-throughput tradeoff," IEEE Transactions on Communications, vol. 58, no. 2, pp. 706$718,2010$.

[24] F. Li, Q. T. Zhang, and S. H. Song, "Efficient optimization of input covariance matrix for MISO in correlated Rayleigh fading," in Proceedings of IEEE Wireless Communications and Networking Conference, March 2007.

[25] K. Huang, R. W. Heath, Jr., and J. G. Andrews, "Uplink SDMA with Limited Feedback: Throughput Scaling," EURASIP Journal on Advances in Signal Processing, special issue on Limited Feedback, vol. 2008, Article ID 479357, 2008.

[26] K. Huang, R. W. Heath, Jr., and J. G. Andrews, "Space Division Multiple Access with a Sum Feedback Rate Constraint," IEEE Trans. on Signal Processing, vol. 55, no. 7, pp. 3879-3891, July 2007.

[27] F. Li and H. Jafarkhani, "Resource allocation algorithms with reduced complexity in MIMO multi- 
International Journal of Computer Science \& Information Technology (IJCSIT) Vol 4, No 2, April 2012

hop fading channels," in Proceedings of IEEE Wireless Communications and Networking

Conference, 2009.

[28] D. J. Ryan, I. B. Collings, I. V. L. Clarkson, and R. W. Heath, Jr., "Performance of Vector Perturbation Multiuser MIMO Systems with Limited Feedback," IEEE Trans. on Communications, vol. 57, no. 9, pp. 2633-2644, Sept. 2009.

[29] F. Li and Q. T. Zhang, "Transmission strategy for MIMO correlated rayleigh fading channels with mutual coupling," in Proceedings of IEEE International Conference on Communications (ICC 2007), June, 2007.

[30] P. Viswanath, D. N. C. Tse, and V. Anantharam, "Asymptotically optimal waterfilling in vector multiple access channels," IEEE Trans. Inform. Theory, vol. 47, pp. 241-267, Feb. 2001.

[31] D. C. Popescu, O. Popescu, and C. Rose, "Interference avoidance for multi access vector channels," in Proc. Int. Symp. Inform. Theory, July 2002, p. 499.

[32] J. Wang and K. Yao, "Multiuser spatio-temporal coding for wireless communications," in Proc. IEEE Wireless Commun. Networking Conf., vol. 1, Mar. 2002, pp. 276C279.

[33] F. Li and H. Jafarkhani, "Multiple-antenna interference cancellation and detection for two users using precoders," IEEE Journal of Selected Topics in Signal Processing, December 2009.

[34] G. G. Raleigh and J.M. Cioffi, "Spatio-temporal coding for wireless communication," IEEE Trans. Commun., vol. 46, pp. 357C366, Mar. 1998.

[35] H. Lu, R. Vehkalahti, C. Hollanti, J. Lahtonen, Y. Hong and E. Viterbo, "New space time code constructions for two-user multiple access channels," IEEE J. Sel. Top. Sign. Proces., vol. 3, no. 6, pp.939-957, Dec. 2009. 